\section{Are we ready for}

\section{endophenotypes in}

attention deficit

\section{hyperactivity disorder?}

\author{
Estamos prontos para
}

os endofenótipos no

\section{transtorno do déficit}

de atenção e

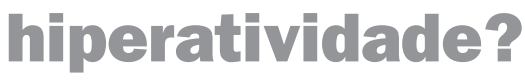

Behavioural genetic studies contrast with molecular genetic studies of Attention Deficit hyperactivity disorder (ADHD). Doyle et al., in their comprehensive review, make clear that heritability estimates from twin studies clearly indicate that there is a strong genetic component in ADHD with heritabilty ranging from 0.6 to $0.9 .{ }^{1}$ In contrast, molecular genetic studies of ADHD have identified non-overlapping chromosomal regions that may contain possible susceptibility genes. This difference between behavioural ratings and molecular findings clearly poses currently a problem for advocates of an endophenotype approach to ADHD.

The term "endophenotype" refers to a phenotype thought to be closer to the biological factors causing a clinical disorder, in contrast to a clinical phenotype, which refers to the clinical signs and symptoms of a syndrome. ${ }^{2}$

The assumption of the endophenotype approach is that it will be possible to identify more easily the neuro-biological factors that underlie the disorder rather than identifying the genetic factors associated with the clinical syndrome. The argument is that the reduced complexity is due to firstly the endophenotype's proximity to the genes thought to produce the disorder and, secondly, to the potential that the endophenotype can better specify the factors that lead to the clinical syndrome. This argument assumes that the endophenotype is better able to discriminate the condition from other conditions and non-cases than the phenotype itself. A further assumption is that the endophenotype truly reflects the pathological processes causing the condition and not epiphenomena associated with the syndrome but which have no causal relation to the clinical condition.

It seems worthwhile at this stage in the field's development to evaluate briefly the success of the endophenotypic approach in better differentiating the ADHD condition from non-cases and associated disorders. The proponents of the approach have used primarily neuropsychological models to define the ADHD endophenotype. A first study employed well-defined neuropsychological tasks, acquired DNA material and contrasted the rigorously defined ADHD sample with well-defined controls. ${ }^{3}$ The phenotypic distinction between the groups differentiated the ADHD from the control group and did so in terms of neuropsychological functioning. Disappointingly, no relationship was found between the endophenotypes: inhibition, response control with the DRD4-7 repeat allele.

Since then an international hunt for a valid endophenotype has commenced to determine the relation between neuropsychological processes and genes. A problem here is that the neuropsychological tasks have identified only 35\%$50 \%$ of combined-type ADHD subjects as exhibiting deficits on processes thought key to the disorder: inhibition, interference control, and processing speed/set shifting. Combining across measures the neuropsychological measures does not substantially increase the number of individuals with impairments. ${ }^{4}$ Hence, a critical weakness of the endophenotype approach is that it employs neuropsychological measures, which do not have the sensitivity nor the specificity to identify to a high degree the clinical phenotype. Differentiation between 
ADHD and disorders such as High Function Autism and Tourette's disorder on neuropsychological measures has proven to be difficult. ${ }^{5}$ Similarly, other neuropsychological measures such as working memory on the basis of a recent meta-analysis show only modest differentiation between the ADHD phenotype and controls. It has been argued that the variability in speed of processing might be the best candidate for endophenotype research. Unfortunately, this measure has found to be associated with High Function Autism to an even greater degree than with ADHD. ${ }^{5}$

In view of this, it is evident that the endophenotype approach has not revealed a short-cut to identifying the causal factors of ADHD. The measures currently used to define the endophenotype are too weak in their sensitivity, lack sufficient specificity and have, currently, no known predictive power for the developmental course of the disorder. This raises the pragmatic question of whether the investment in the endophenotype is worth it at this point in time? Would the field not be better to take stock and identify the neuropsychobiological processes that differentiate ADHD from controls, accounts for its subtypes and associated disorders as well as from syndromes with putative neuropsychological deficits proposed to account for ADHD? Without this first step being achieved one may wonder what is the gain in endophenotypes, when the phenotype relationship to neuro-biological processes is unclear.

Joseph Sergeant Clinical Neuropsychology, Vrije Universiteit, Amsterdam

\section{References}

1. Doyle AE, Willcutt EG, Seidman LJ, Biederman J, Chouinard VA, Silva J, et al. Attention-deficit/hyperactivity disorder endophenotypes. Biol Psychiatry. 2005;57(11):1324-35.

2. Gottesman II, Gould TD. The endophenotype concept in psychiatry: etymology and strategic intentions. Am J Psychiatry. 2003;160(4):636-45.

3. Swanson J, Oosterlaan J, Murias M, Schuck S, Flodman P, Spence $M A$, et al. Attention deficit/hyperactivity disorder children with a 7repeat allele of the dopamine receptor D4 gene have extreme behavior but normal performance on critical neuropsychological tests of attention. Proc Natl Acad Sci USA. 2000;97(9):4754-9.

4. Nigg JT, Willcutt EG, Doyle AE, Sonuga-Barke EJ. Causal heterogeneity in attention-deficit/hyperactivity disorder: do we need neuropsychological subtypes? Biol Psychiatry. 2005;57(11):1224-30.

5. Geurts HM, Verté S, Oosterlaan J, Roeyers H, Sergeant JA. How specific are executive functioning deficits in Attention Deficit hyperactivity disorder and autism? J Child Psychol Psychiatry. 2004;45(4):836-54. 